\title{
L'arrivée de l'échographie pratiquée au point de service, plus précisément au service des urgences, au Canada
}

\author{
Paul Atkinson
}

Au printemps de 2012, l'Association canadienne des médecins d'urgence (ACMU) a publié une importante déclaration de position révisée dans laquelle elle se prononçait en faveur de l'échographie pratiquée au point de service (EPS) par les urgentologues au service des urgences $(\mathrm{SU}){ }^{1}$

L'échographie au service des urgences a vu le jour au Canada, en 2001: l'échocardiographie au service des urgences était le fruit du mouvement international en faveur de l'échographie à l'urgence, dont les origines remontent à la fin des années 1980, aux Etats-Unis. ${ }^{2}$ La Société canadienne d'échographie au département d'urgence (SCÉDU) a été la seule organisation à délivrer un certificat officiel en matière d'échographie au service des urgences, au Canada, au cours de la dernière décennie. L'Association est favorable à l'utilisation accrue de l'échographie au service des urgences, au Canada, et demande de déployer de nouveaux efforts pour que tous les urgentologues puissent acquérir cette nouvelle compétence.

Les définitions de l'échographie au service des urgences peuvent varier selon les indications cliniques, mais la plupart des urgentologues diront qu'il s'agit d'un examen diagnostique ou interventionnel, effectué et interprété par un urgentologue, au cours de la première rencontre avec un patient, afin d'évaluer des problèmes urgents. ${ }^{1}$

Le recours à l'EPS comme examen complémentaire en médecine d'urgence (MU) est maintenant une pratique bien établie, tant à l'échelle nationale qu'à l'échelle internationale. Au début, les données à l'appui du recours à l'EPS provenaient d'expériences de traitement de patients qui avaient subi un traumatisme contondant. ${ }^{3}$ Toutefois, son champ de pratique s'est élargi à mesure que les urgentologues faisaient face à d'autres problèmes cliniques pour lesquels l'EPS pouvait se montrer utile dans les évaluations et les interventions. Outre les traumas, les applications de base comprennent aujourd'hui la recherche d'un anévrisme de l'aorte abdominale, une échocardiographie « focalisée » en cas d'arrêt cardiaque et la confirmation d'une grossesse intra-utérine au cours du premier trimestre. Puis, avec le temps, l'éventail de réponses fournies par l'EPS s'est élargi et il inclut maintenant des problèmes comme la recherche d'un épanchement pleural ou d'un pneumothorax, la recherche d'une thrombose veineuse profonde, l'évaluation de la veine cave inférieure pour déterminer l'état de remplissage, l'application de protocoles en cas de choc, ainsi que des indications plus courantes comme la cholélithiase et l'hydronéphrose. ${ }^{1}$ Par ailleurs, l'échoguidage rend plus sûre la mise en place de cathéters veineux centraux et accroît le taux de réussite des blocs nerveux régionaux. ${ }^{4-7}$

Dans la pratique quotidienne, les urgentologues font face souvent à des cas difficiles et ils tentent d'identifier des maladies graves ou de réduire le risque de complications liées à des interventions, et ce, en temps opportun. « Nécessité est mère de l'invention », diton; aussi les urgentologues continuent-ils à trouver de nouvelles applications et à élargir les indications de l'EPS. Point à souligner: l'American College of Emergency Physicians (ACEP) a divisé dernièrement

Tiré de Emergency Department, Saint John Regional Hospital, Saint John, NB.

Correspondance à: Dr. Paul Atkinson, Emergency Department, Saint John Regional Hospital, University Avenue, Saint John, NB E2L 4L4; Paul. Atkinson@dal.ca, paul.atkinson@horizonnb.ca.

Cet article a été évalué par les pairs.

(c) Association canadienne des médecins d'urgence 
l'échographie au service des urgences en cinq catégories cliniques fonctionnelles afin de clarifier ses diverses utilisations:

- Réanimation: échographie utilisée directement en lien avec la phase aiguë de la réanimation

- Diagnostic: échographie utilisée comme nouvel outil d'imagerie diagnostique

- Signe ou symptôme: échographie utilisée dans le cadre d'un cheminement clinique, fondé sur les signes ou symptômes (ex.: essoufflement)

- Guidage: échographie utilisée comme aide au guidage interventionnel

- Traitement et surveillance: échographie utilisée comme outil de traitement ou de surveillance des fonctions physiologiques ${ }^{8}$

Point digne de mention, l'EPS ne remplace pas la pratique courante de l'échographie; elle consiste plutôt en un examen restreint d'une région, généralement effectué au chevet du malade, dans des conditions non idéales, et circonscrit dans le temps. L'application vise donc à répondre à une question (ou à une série de questions) d'ordre clinique, susceptible de compléter les soins cliniques. Par ailleurs, la pratique comme telle de l'EPS a gagné du terrain dans des domaines qui ne relevaient pas autrefois de l'échographie, notamment le diagnostic de certaines affections pulmonaires, par exemple la présence de liquide interstitiel ou alvéolaire. ${ }^{9}$ À mesure que l'échographie progressait en MU et que ses utilisateurs établissaient des liens avec des médecins dans d'autres champs de pratique, l'examen a été désigné sous différentes appellations: échographie ciblée au service des urgences, échographie en soins urgents et très urgents, échographie 《focalisée » à l'urgence, et maintenant EPS, acronyme généralement connu. ${ }^{9}$

L'EPS a maintenant franchi les frontières entre spécialités, a de la pertinence aux yeux de la plupart des groupes de cliniciens et est en voie de faire partie intégrante du programme d'études de premier cycle en médecine dans certains centres. ${ }^{9,10}$ L'engouement pour ce type d'examen s'explique aussi par l'amélioration et l'adaptabilité des appareils d'échographie, notamment par la diminution du prix et de la grosseur des machines, une amélioration de la qualité et une portabilité accrue, la convivialité, et la réduction du temps de mise en route. Une invitation est lancée à tous les médecins pour qu'ils prennent connaissance de la liste des applications actuelles de l'EPS, dressée par Moore et Copel, dans différentes spécialités cliniques. ${ }^{9}$
Compte tenu de la diversité d'application de l'EPS, il faut veiller à ce que l'examen soit pratiqué dans le respect des principes de la bonne gouvernance.

Maintenant que la pratique de l'échographie a acquis ses « lettres de noblesse », il est important que la collectivité de praticiens en échographie au service des urgences au Canada envisage soigneusement les prochaines étapes de son évolution. Comme le Collège royal des médecins et chirurgiens du Canada (CRMCC), le Collège des médecins de famille du Canada (CMFC), la SCÉDU, et l'ACMU recommandent d'inscrire la formation en échographie au programme de base, il est essentiel que les possibilités de formation en EPS fassent partie intégrante de tous les programmes de résidence, au pays.

Actuellement, la formation en EPS dans les programmes canadiens de résidence en MU varie énormément, et ce, tant sur le plan du degré de connaissances que sur celui de la qualité; en effet, la plupart des résidents ont recours à des ressources didactiques extérieures au programme de résidence pour compléter leurs connaissances en échographie. ${ }^{11}$ Aussi une normalisation rigoureuse de la formation dans tous les programmes de résidence s'impose-t-elle, de sorte à rendre caduque l'obligation d'acquérir un certificat supplémentaire en EPS, outre le titre de FRCPC (MU) ou le Certificat du Collège des médecins de famille du Canada (CCMF [MU]). Ainsi, l'acquisition des compétences en EPS serait considérée au même titre que les autres compétences de base en MU, comme l'assistance respiratoire, l'électrocardiographie, et l'interprétation des radiographies, pour lesquelles il n'est plus nécessaire maintenant d'obtenir un certificat supplémentaire.

Pour ce faire, il faut tenir compte des points suivants:

- Formation. Bien qu'il soit possible de poursuivre, dans un avenir prévisible, l'enseignement selon le modèle classique d'un cours d'introduction ou de modules, suivis de la réalisation d'un certain nombre d'examens par échographie, réalisés sous supervision, l'adoption d'une approche à facettes s'impose pour les raisons suivantes: certains états pathologiques sont peu fréquents; les superviseurs formés peuvent se faire rares dans certaines régions, et la supervision individuelle n'est pas toujours possible. D'autres modèles de formation pourraient comprendre l'apprentissage par simulation ou sur le Web, deux formules qui sont en voie de s'implanter et qui gagnent du terrain. ${ }^{12,13}$ L'acquisition de compétences 
en EPS est un aspect très important de la formation clinique des urgentologues, et la réussite du stage en MU devrait comporter pour tous les résidents l'acquisition obligatoire d'un ensemble minimal de compétences..$^{14,15}$ La formation en échographie donnée aux résidents en $M U$ devrait être structurée de manière que ceux-ci puissent intégrer l'échographie dans la pratique clinique quotidienne. ${ }^{12}$

- Délivrance de titres et certificats. Il est essentiel qu'un stagiaire en EPS fasse la preuve de sa compétence dans une application particulière avant de pratiquer seul. D'après certains, la réalisation d'un grand nombre d'examens par échographie peut laisser supposer l'acquisition de la compétence en la matière, données à l'appui, mais, dans la pratique courante, les examens inscrits ne sont pas tous de même qualité. ${ }^{8}$ Par contre, d'autres préconisent l'amorce d'un processus d'évaluation de la compétence après une période recommandée de formation. Ce type d'évaluation se réalise en fonction d'un certain nombre de critères objectifs et est moins tributaire du nombre d'examens inscrits que l'autre formule. Etre suffisamment compétent pour pratiquer ne veut pas dire tout simplement être capable d'effectuer l'examen mais aussi, et surtout, être capable de comprendre la portée des résultats en fonction de la pratique clinique..$^{17,18} \mathrm{Il}$ faudrait veiller à ce que la délivrance du certificat de compétence, dans le cadre des programmes d'obtention du diplôme en MU au Canada, soit par la voie du FRCPC (MU) ou celle du CCMF (MU), vise au moins toutes les applications de base de l'EPS. ${ }^{1}$

- Gouvernance. Une fois devenus praticiens indépendants, les urgentologues doivent avoir le souci de garder leur compétence à jour. Le manque de pratique d'une application particulière peut aboutir à la perte de cette compétence. Les évaluations par les pairs et les audits jouent un rôle important dans la démonstration du maintien de la compétence. Il est essentiel de connaître les derniers résultats de la recherche médicale en la matière. L'entretien périodique du matériel et l'évaluation de l'assurance de la qualité des examens par échographie sont des éléments indispensables.

- Recherche. Certes, de plus en plus de données probantes confirment les avantages de l'EPS en médecine d'urgence, mais une grande partie de la documentation recueillie jusqu'à maintenant ne fournit pas la preuve d'un avantage certain de l'examen sur le plan des résultats cliniques. ${ }^{8} \mathrm{Il}$ est important de se soumettre aux exigences des études bien conçues pour renforcer la pertinence de la pratique. Les organisations médicales nationales en MU et en soins intensifs devraient prendre l'initiative en offrant les possibilités de réseautage nécessaires à l'élaboration d'études prospectives bien conçues et menées sur une grande échelle ainsi qu'à la constitution de bases de données.

- «Fellowship ». Dans des pays comme les États-Unis, de nombreux « fellowships » en EPS s'offrent aux urgentologues. ${ }^{19}$ L'établissement d'un réseau comparable de «fellowships» en EPS au Canada, doté d'un programme d'études national, fondé sur l'évaluation des besoins, serait un gage d'excellence à venir en échographie au service des urgences pour les responsables, les chercheurs et les formateurs.

- Relations internationales. L'EPS continue à évoluer à l'échelle internationale et, pour cette raison, il importe que le Canada fasse partie de la collectivité internationale à l'origine de cette croissance. Des organisations telles que l'International Federation of Emergency Medicine (IFEM) et WINFOCUS organisent des groupes de discussion dans lesquels membres du corps professoral et stagiaires peuvent faire part de leurs connaissances et de leurs compétences. Il est probable qu'avec le temps un programme international et une norme de pratique voient le jour. Les chefs de file en matière d'échographie au service des urgences au Canada doivent donc s'engager à travailler en étroite collaboration avec des collègues de partout dans le monde à l'élaboration de cette norme.

Pour le moment, la collectivité devrait se réjouir de l'arrivée de l'échographie au service des urgences, au Canada. Elle doit aussi reconnaître que le champ de l'EPS s'élargit tant au sein de sa spécialité qu'au sein d'autres spécialités et, de ce fait, il faut veiller à la formation appropriée en EPS de tous les médecins qui doivent prendre des décisions rapides en matière de soins cliniques, dans les cas où l'efficacité de l'échographie est déjà avérée.

Mots clés: délivrance de titres et certificats, échographie, échographie pratiquée au point de service, programme d'études de premier cycle en médecine

Intérêts concurrentiels: Aucun déclaré. 


\section{RÉFÉRENCES}

1. Henneberry RJ, Hanson A, Healy A, et al. Use of point of care sonography by emergency physicians. Canadian Association of Emergency Physicians position statement. CFEM 2012;14:106-12.

2. Jehle D, Davis E, Evans T, et al. Emergency department sonography by emergency physicians. Am $\mathcal{f}$ Emerg Med 1989;7:605-11, doi:10.1016/0735-6757(89)90283-0.

3. Boulanger BR, McLellan BA, Brenneman FD, et al. Prospective evidence of the superiority of a sonography based algorithm in the assessment of blunt abdominal injury. If Trauma 1999;47:632-37, doi:10.1097/00005373199910000-00005.

4. Making bealth care safer: a critical analysis of patient safety practices. Prepared for: Agency for Healthcare Research and Quality. Prepared by: University of California at San Francisco (UCSF) - Stanford University Evidence-based Practice Center. Available at: www.ahrq.gov (accessed March 18, 2013).

5. Atkinson P, Boyle A, Robinson S, Campbell-Hewson G. Should ultrasound guidance be used for central venous catheterization in the emergency department? Emerg Med 7 2005;22:158-64, doi:10.1136/emj.2003.011288.

6. Leung J, Duffy M, Finckh A. Real-time ultrasonographically guided internal jugular vein catheterization in the emergency department increases success rates and reduces complications: a randomized, prospective study. Ann Emerg Med 2006;48:540-7, doi:10.1016/j.annemergmed.2006.01.011.

7. Gray A. Ultrasound-guided regional anaesthesia: current state of the art. Anesthesiology 2006;104:368-73, doi:10.1097/ 00000542-200602000-00024.

8. American College of Emergency Physicians. Emergency ultrasound guidelines 2008. Available at: http://www.acep.org (accessed March 18, 2013).
9. Moore CL, Copel JA. Point of care ultrasonography. $N$ Engl 7 Med 2011;364:749-57, doi:10.1056/NEJMra0909487.

10. Wong I, Jayatilleke T, Kendall R, Atkinson P. Feasibility of a focused ultrasound training programme for medical undergraduate students. Clin Teach 2011;8:3-7, doi:10. 1111/j.1743-498X.2010.00416.x.

11. Kim DJ, Theoret J, Woolfrey K, Kendall JL. Experience with emergency ultrasound training by Canadian emergency medicine residents: a national survey. CFEM 2012;14:S1.

12. Sidhu HS, Olubaniyi BO, Bhatnagar G, et al. Role of simulation-based education in ultrasound practice training. 7 Ultrasound Med 2012;31:785-91.

13. Filippucci E, Meenagh G, Epis O, et al. E-learning in ultrasonography: a web-based approach. Ann Rheum Dis 2007;66:962-5, doi:10.1136/ard.2006.064568.

14. Heller M, Mandavia D, Tayal V, et al. Residency training in emergency ultrasound: fulfilling the mandate. Acad Emerg Med 2002;9:835-9, doi:10.1111/j.1553-2712.2002.tb02174.x.

15. Reardon R, Heegaard B, Plummer D, et al. Ultrasound is a necessary skill for emergency physicians. Acad Emerg Med 2006;13:334-6, doi:10.1111/j.1553-2712.2006.tb01702.x.

16. Lanoix R, Leak LV, Gaeta T, et al. A preliminary evaluation of emergency ultrasound in the setting of an emergency medicine training program. Am 7 Emerg Med 2000;18:41-5, doi:10.1016/S0735-6757(00)90046-9.

17. Canadian Emergency Ultrasound Society. CEUS recommended standards. Available at: http://ceus.ca/008-position_statements/ 008-01.advanced_applications.htm (accessed March 18, 2013).

18. College of Emergency Medicine Ultrasound Subcommittee. Emergency medicine ultrasound, level 1 training. Available at: http://www.collemergencymed.ac.uk/Training-Exams/Training/ Ultrasound\%20training/default.as (accessed March 18, 2013).

19. EUS fellowships. Available at: http://eusfellowships.com/ programs.php (accessed March 18, 2013). 